\title{
The Effect of Bangerter Occlusion Foils on Blepharospasm and Hemifacial Spasm in Occlusion-Positive and Occlusion-Negative Patients
}

\author{
Raman Malhotra ${ }^{*}, 1$ Siew-Yin Then ${ }^{1}$, Alison Richards ${ }^{1}$ and Elizabeth Cheek $^{2}$ \\ ${ }^{1}$ Corneoplastic Unit, Queen Victoria Hospital NHS Foundation Trust, Holtye Road, East Grinstead, West Sussex RH19 \\ $3 D Z, U K$ \\ ${ }^{2}$ School of Computing, Mathematical and Information Sciences, University of Brighton, Lewes road, Brighton BN2 4AT, \\ $U K$
}

\begin{abstract}
Objective: To test the hypothesis that occlusion-positive (OP) patients with blepharospasm (BEB) or hemifacial spasm (HFS) will benefit from a Bangerter occlusion foil (BOF), compared to occlusion-negative (ON) patients. OP/ON was based on immediate improvement in spasm with placement of a hand in front of either eye.
\end{abstract}

Design: Prospective non-randomised single-centre pilot study.

Participants: Fifteen-patients (6 BEB, 9 HFS).

Methods: Patients were identified as OP or ON and wore highest-density BOF tolerable over one spectacle lens for 1 month. Outcomes were assessed at 1 month.

Main Outcome Measures: Validated quality-of-life questionnaire (CDQ-24), scores of blink-rate and spasm severity assessed by two observers from video-recordings.

Results: OP group had mean improvement in all scores. There was no change or worsening of scores in the ON group. In both BEB and HFS, more OP patients reported subjective benefit from wearing a foil (2 of 4 BEB, and 2 of 2 HFS) compared to the ON group ( 0 of $2 \mathrm{BEB}$, and 1 of $7 \mathrm{HFS}$ ).

Conclusion: OP patients with BEB and HFS are more likely to experience improvement in spasms from wearing a BOF compared to ON patients. The occlusion test should be considered on all patients with BEB or HFS.

Keywords: Essential blepharospasm, bangerter occlusion foils, occlusion test.

\section{INTRODUCTION}

Benign essential blepharospasm (BEB) is characterised by bilateral involuntary spasmodic contraction of the eyelid protractors where aetiology is not known. Hemifacial spasm (HFS), in contrast is usually unilateral with an identifiable underlying cause, including a vascular loop or tumor compression of the facial nerve [1-3].

Botulinum toxin (BT) and surgical extirpation of eyelid protractor muscles are the most effective treatments available [1-7]. Most patients with blepharospasm benefit from reduction of sensory triggers, particularly light, and tinted lenses can significantly reduce spasm in some patients [811 ]. Nearly $80 \%$ of patients with BEB report that bright light triggers or exacerbates their symptoms [3], and patients with BEB have been shown to be more light-sensitive than controls [8].

Bangerter occlusion foils (BOF) are translucent, plastic filters that adhere to the front of a spectacle lens. They block light transmission and reduce visual acuity in a graded fashion. We previously reported a case of facial palsyinduced bilateral blepharospasm, which improved with the

*Address correspondence to this author at the Corneoplastic Unit, Queen Victoria Hospital, Holtye Road, East Grinstead, RH19 3DZ, UK; Tel: 01342 414549; Fax: 01342 414106; E-mail: raman.malhotra@qvh.nhs.uk placement of a hand a few centimetres in front of either eye ("occlusion-positive") [12]. The subsequent placement of a BOF over one spectacle lens has allowed this patient to remain asymptomatic without the need for any other treatments, including BT injections.

We have since observed a similar reduction in symptoms using the BOF in other occlusion-positive $(\mathrm{OP})$ patients with BEB and curiously also with HFS. The aim of this pilot study was to test the hypothesis that the BOF improves symptoms of BEB and HFS in OP patients, but not occlusion-negative $(\mathrm{ON})$ patients, helping establish the clinical utility of the occlusion-sign in identifying patients most likely to benefit from the BOF.

\section{METHODS}

\section{Patients}

This was a prospective non-randomised interventional pilot study approved by the Brighton West Research Ethics Committee and the Research and Development Committee of the Queen Victoria Hospital NHS Foundation Trust, East Grinstead, United Kingdom. Informed consent was obtained from all subjects prior to enrolment in the study.

Subjects with BEB or HFS were recruited from the Corneoplastic Unit Facial Dystonia Clinic. BEB was defined as involuntary spasmodic contractions of the eyelids not 
associated with any known aetiology. HFS was defined as unilateral involuntary tonic-clonic contractions of the muscles innervated by the ipsilateral facial nerve. Of a total of 41 patients attending the Facial Dystonia clinic, 21 eligible patients with BEB or HFS were identified. Sixteen patients agreed to initially participate, but one patient subsequently withdrew as she elected to have BT treatment one week into the study. Fifteen patients successfully completed the study, of which 9 had HFS and 6 had BEB. Five patients were unable to or refused to participate, including one patient with $\mathrm{BEB}$ who had tried the BOF previously without effect. Patients with secondary blepharospasm, predominantly monocular vision, or prone to falls were excluded from the study.

\section{Study Visits}

Patients attended two study visits, at the beginning and end of a one-month period of wearing a Bangerter occlusion foil (BOF). To reduce the potential confounding effect of concurrent BT therapy, which the majority of participants were also receiving, the first study visit and commencement of BOF use was timed to occur just prior to the next scheduled BT injection, thus ensuring a maximal time interval from last injection. BT injections were therefore delayed on occasion by up to a month in a few patients. Patients were encouraged to continue using any other treatments for their dystonia, including tinted spectacles, during the study period.

\section{Data Collection}

At the first (pre-intervention) study visit, all patients were assessed by the same ophthalmologist (ST). Baseline data included: age, gender, date of diagnosis of idiopathic facial dystonia, previous and current treatments, presence of other movement disorders, past ophthalmic and medical history, current medications, time from last BT treatment, current level of visual acuity and findings from a full ophthalmic examination to exclude secondary causes of blepharospasm.

Patients were identified as occlusion-positive (OP) or occlusion-negative $(\mathrm{ON})$ depending on whether there was an immediate improvement in eyelid spasm with the placement of an examiner's hand or occluder approximately $3 \mathrm{~cm}$ in front of either eye, avoiding any direct facial or spectacle contact (Fig. 1a, b). Such an improvement was judged
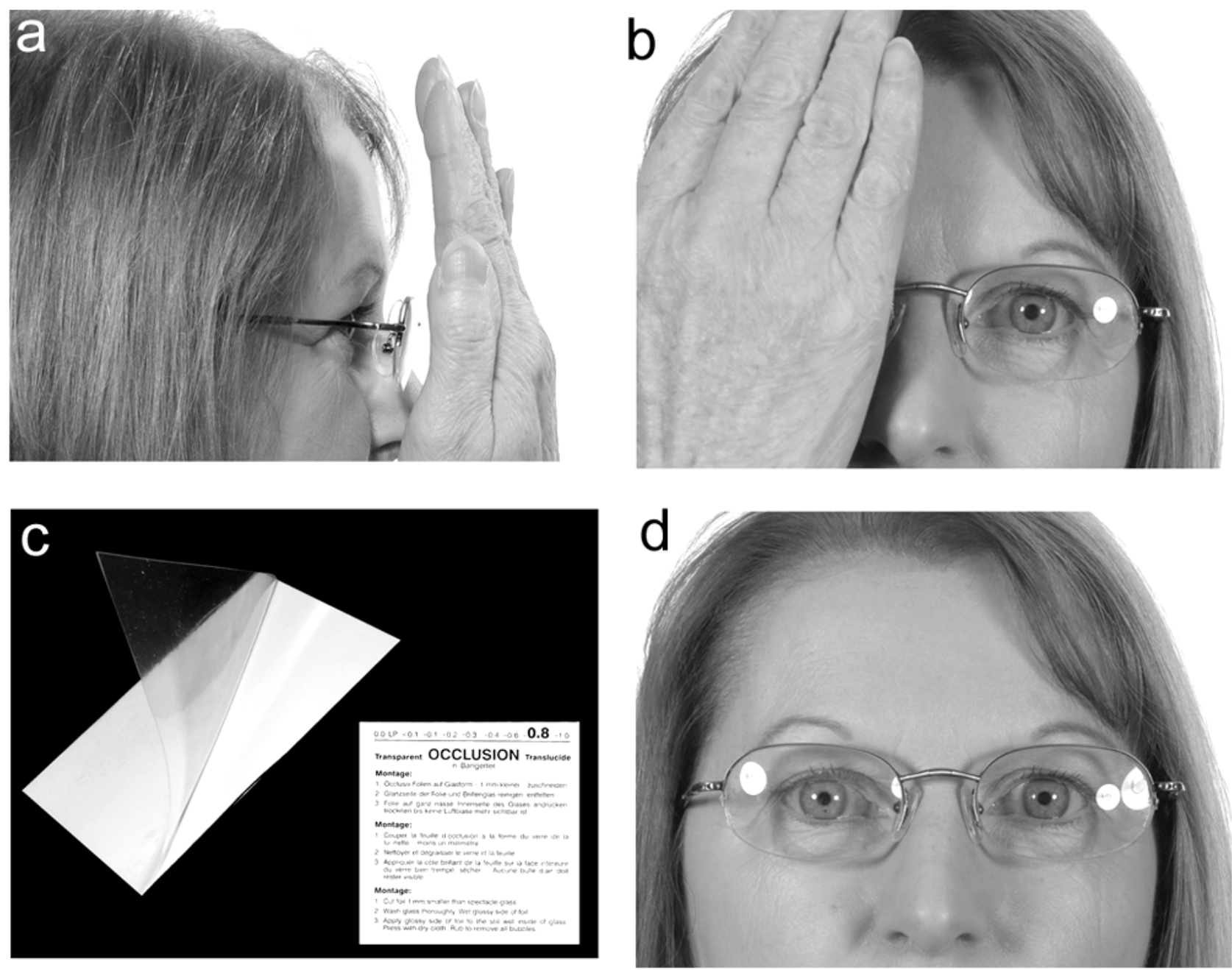

Fig. (1). (a, b) Occlusion test is performed with patient's hand placed $3 \mathrm{~cm}$ in front of the ipsilateral eye, avoiding facial or spectacle contact. (c) The Bangerter occlusion foil is a disposable self-adhesive foil, which can be cut to size to fit over a spectacle lens. 0.8 density foil shown here. (d) Bangerter occlusion foil (0.2 density) placed over right spectacle lens. 
subjectively both by the examiner and also the patient and was defined as a reduction of at least $30 \%$ in frequency and severity of spasm. Patients from both the OP and ON groups were then fitted by an orthoptist with a single BOF (Haag Streit UK Ltd, Harlow Essex, UK) over one spectacle lens (Fig. 1c, d). The system of BOF and expected visual acuity for each density of foil is outlined in Table $\mathbf{1}$. The highestdensity BOF that was visually-tolerable for the patient was used, and patients were given a choice as to which eye they wished to wear the BOF over. Patients who did not regularly wear spectacles were provided with clear frames, whilst those who had separate reading and distance spectacles were given a separate BOF for each pair of glasses. Patients were asked to wear the BOF for a one-month period, at the end of which they attended their second (post-intervention) study visit.

Table 1. Bangerter Occlusion Foils -Grading of Foil and Expected Visual Acuity

\begin{tabular}{|c|c|}
\hline Density of Foil & Snellen Visual Acuity \\
\hline \hline 1.0 & $20 / 20$ \\
\hline 0.8 & $20 / 25$ \\
\hline 0.6 & $20 / 30$ \\
\hline 0.4 & $20 / 40$ \\
\hline 0.3 & $20 / 80$ \\
\hline 0.2 & $20 / 100$ \\
\hline 0.1 & $20 / 200$ \\
\hline$<0.1$ & $<20 / 300$ \\
\hline LP & Light Perception \\
\hline 00 & No light \\
\hline
\end{tabular}

\section{Outcome Measures}

The effect of wearing the BOF on facial dystonia over the one-month intervention period was measured with a quality-of-life questionnaire (QOL) and video-recordings of patients, conducted at both the first and second study visits.

The CDQ-24 questionnaire, a validated disease-specific QOL designed to assess the level of impairment resulting from a patient's craniocervical dystonia, has been shown to be both reliable and sensitive in measuring treatmentinduced changes over a 4-week and 1-year period [13]. The 24-item questionnaire is based on five sub-scales that address stigma, emotional well-being, pain, activities of daily living and social/family life, with each item consisting of five statements representing increasing severity of impairment, scored from 0 to 4 . Patients completed the same questionnaire at the first and second study visits, and at the latter, were asked to record their level of impairment during the one-month intervention period. Change in total score as well as scores for each of the five subscales between the first and second study visit questionnaires were assessed for each patient.

Video-recordings of patients over an uninterrupted 2minute interval were also taken at pre and post-intervention study visits. At the second study visit, patients were recorded with the BOF being worn. The tapes were viewed at a later date by two independent observers, one masked and one unmasked to the occlusion-status of the patients, and the blink-rate and frequency and severity of involuntary movements were scored in a method similar to that described by Vitale et al. [14]. The number of blinks was counted over the 2-minute interval. The frequency of involuntary movements was scored from $0-3$ in the following order: no involuntary movement (0), movement present $<10 \%$ of time (1), movement present $>10 \%$ of time (2) and movement present constantly (3). The severity of involuntary movement was scored from $0-3$ in the following order: no involuntary movement (0), increased blinking and/or mild involuntary facial movement (1), forced closure of eyelids and/or moderate involuntary facial movement (2) and severe forced closure of eyelids and/or severe involuntary facial movement (3).

Blink-rate and scores used for analysis was taken as the average of the two video observers' scores. Changes in blink-rate and scores between the first and second study visit were assessed.

\section{Statistical Analysis}

Differences between pre- and post-intervention scores for CDQ-24 questionnaires and video-ratings were calculated for each patient. Since the differences were not normally distributed, the non-parametric Mann-Whitney test was used to compare the occlusion-positive and occlusion-negative groups. A two-tailed 5\% significance level was used throughout. The analysis was carried out using SPSS version 14 software (SPSS Inc. Chicago).

\section{RESULTS}

Fifteen-patients ( 6 with BEB, 9 with HFS) completed the study. Six ( $40 \%$ ) patients (5 female, 1 male) were occlusionpositive (OP) and 9 (5 female, 4 male) were occlusionnegative (ON). Of the 9 patients with HFS, 2 were OP and 7 were ON. Of the 6 with BEB, 4 were OP and 2 were ON. Mean age was 63.3 years (range $44-80$ years) and 73.5 years (range 43-93 years) for the OP and ON groups, respectively.

\section{Previous/Concurrent Treatments}

14 of the 15 patients were receiving regular BT injections at time of enrolment. 1 patient in the OP group, who had already been successfully using the BOF prior to enrolment in the study, had not required BT injections for three years. All patients $(100 \%, 14 / 14)$ commenced the study at least 4 weeks after their last BT injection, with the median time from last injection being 12 weeks (range 4 weeks-36 weeks) in the OP group and 15 weeks (range 4-42 weeks) in the ON group.

All patients had tried tinted spectacles with varying success. One patient found pinhole spectacles beneficial in relieving blepharospasm. Three patients (1 OP and $2 \mathrm{ON}$ ) had undergone previous limited orbiculectomy surgery.

\section{BOF Use}

The majority of patients in both groups $(80 \%$ OP, $67 \%$ $\mathrm{ON}$ ) wore the BOF full-time (all waking hours). The remaining patients elected not to wear the BOF whilst at work, or only at certain times of the day, and in these 
Table 2. Mean Change (Pre-Intervention Minus Post-Intervention) in Outcome Scores

\begin{tabular}{|l|c|c|c|}
\hline \multicolumn{1}{|c|}{ Mean Change } & Occlusion-Positive & Occlusion-Negative & $\begin{array}{c}\text { Exact Significance of Difference Between OP and ON Groups } \\
\text { (Mann-Whitney Test) }\end{array}$ \\
\hline \hline CDQ24 Total Score & 7.2 & -2.7 & 0.147 \\
\hline a. Stigma & 3.6 & -0.6 & 0.147 \\
\hline b. Emotional & 1.2 & 0.2 & 0.518 \\
\hline c. Pain & 1.4 & 0.0 & 0.147 \\
\hline d. ADL & 1.0 & -2.7 & 0.438 \\
\hline e. Social & 0.0 & 0.3 & 0.699 \\
\hline Blink-Rate & 39.8 & -20.4 & 0.029 \\
\hline Frequency of Movement & 1.2 & 0.3 & 0.240 \\
\hline Severity of Movement & 1.4 & -0.1 & 0.019 \\
\hline
\end{tabular}

$\mathrm{a}-\mathrm{e}=$ the five subscales of the 24-item CDQ-24 QOL.

NB. A positive mean indicates that the post-treatment value is lower than the pre-treatment value (improvement), whilst a negative mean indicates that the post-treatment value is higher than the pre-treatment value (worsening).

patients the time of use ranged from 4-8 hours per day. The mean strength of BOF worn was 0.45 (range 0.3-0.6) in the OP group and 0.58 (range $0.4-0.8$ ) in the ON group.

\section{Outcomes}

There was a difference in the mean change from pre to post-intervention scores between OP and ON groups (Table 2). There was a positive mean change (improvement) between pre and post-intervention scores for total CDQ-24 score, frequency and severity of involuntary movement score, and blink-rate in the occlusion-positive group. In contrast, there was no change or a negative mean change (worsening) of these scores and blink-rate in the occlusionnegative group. This difference in mean change between the OP and ON groups was statistically significant for blink-rate $(\mathrm{p}=0.029)$ and severity of $\operatorname{spasm}(\mathrm{p}=0.019)$.

Of the 9 patients with HFS, those who were OP (2) reported a subjective benefit from the Bangerter foil. Of those who were ON (7), 1 reported a subjective benefit, whilst the remaining 6 did not report any benefit from wearing the foil.

Of the 6 patients with BEB, 4 who were OP reported a subjective benefit from wearing the foil, whilst 2 did not report any subjective benefit. None of the patients who were ON (2) reported any subjective benefit (Table 3 ).

\section{Complications of BOF Use}

There were no significant reported complications from patients in either group from wearing the BOF.

\section{DISCUSSION}

The results of this pilot study support our hypothesis that occlusion-positive patients with BEB or HFS are more likely to experience a reduction in their symptoms from wearing the BOF, than those who are occlusion-negative. When measured subjectively with the CDQ-24 QOL, and objectively with observation of blink-rate, frequency and severity of spasm, OP patients showed a statistically significant difference in improvement for blink-rate and severity of blepharospasm compared to ON patients.
Anderson et al. [3] have suggested a simple conceptual approach to the multiple factors underlying the origin and manifestations of BEB. They propose that blepharospasm is due to a defective neurological circuit rather than a specific defective locus. Afferent stimuli, such as light and ocular irritation, are transmitted via the optic and trigeminal nerves to an as-yet-unidentified control centre, which may be located in the basal ganglia, midbrain or brainstem. Dysfunction of this centre results in failure of modulation of efferent signals transmitted by the facial nerve to the eyelid protractors, leading to involuntary eyelid contracture. This aberrant cycle becomes self-perpetuating as the resultant eyelid spasms create further irritation of the eyes, which will act as sensory stimuli [3]. Sensory triggers for BEB include bright light and ocular irritation [3, 8, 16]. Both FL41-tinted and grey-tinted lenses can significantly improve blepharospasm $[9,11]$.

Perhaps more curious is the finding of occlusionpositivity and the effect of the BOF in patients with HFS. HFS has traditionally been considered to be due to an identifiable underlying condition affecting the facial nerve. Furthermore, just as in facial palsy-induced blepharospasm, where in an isolated case we first found the BOF to be effective, patients with HFS also tend not to be lightsensitive or photophobic [12]. Interestingly, prior facial-palsy has been recognised in up to 1 in 4 cases of HFS and perhaps electrophysiology may play a more valuable role in identifying such patients and possibly helping further understand the basis of occlusion-positivity $[15,16]$. However, we have also anecdotally observed that some patients with HFS experience reduction in their lower facial as well as eyelid spasm from wearing FL-41 tinted spectacle lenses. We believe that the role and thus modulation of sensory stimuli in HFS therefore remains an area that requires further study.

The BOF degrades visual acuity by reducing light transmission, and may improve blepharospasm by reducing noxious light or other complex visual stimuli mediated by the trigeminal and optic nerves into the aberrant blink cycle. All patients in the study had used tinted lenses with variable success. As no measurements of light-sensitivity were made, 
Table 3. Individual Changes (Pre-Intervention Minus Post-Intervention) in Outcome Scores

\begin{tabular}{|c|c|c|c|c|c|c|c|c|c|}
\hline Patient & $\begin{array}{c}\text { CDQ24 } \\
\text { Total Score }\end{array}$ & a. Stigma & b. Emotional & c. Pain & d. ADL & f. Social & $\begin{array}{l}\text { Blink } \\
\text { Rate }\end{array}$ & $\begin{array}{c}\text { Frequency of } \\
\text { Movement }\end{array}$ & $\begin{array}{l}\text { Severity of } \\
\text { Movement }\end{array}$ \\
\hline \multicolumn{10}{|c|}{ Occlusion-Positive Patients } \\
\hline $1 \mathrm{HFS}$ & 3 & 0 & 1 & 0 & 2 & 0 & -32 & 2 & 1 \\
\hline $2 \mathrm{HFS}$ & 2 & 4 & 0 & 0 & -3 & 1 & 17 & 1 & 1 \\
\hline $3 \mathrm{BEB}$ & 36 & 9 & 4 & 7 & 11 & 5 & 125 & 3 & 3 \\
\hline $4 \mathrm{BEB}$ & -14 & -1 & -5 & 0 & -4 & -4 & 12 & 0 & 0 \\
\hline $5 \mathrm{BEB}$ & 11 & 4 & 4 & 0 & 4 & 2 & 38 & 1 & 1 \\
\hline $6 \mathrm{BEB}$ & 9 & 6 & 6 & 0 & -1 & -2 & 77 & 0 & 2 \\
\hline \multicolumn{10}{|c|}{ Occlusion-Negative Patients } \\
\hline $7 \mathrm{BEB}$ & -4 & -2 & 1 & 8 & -11 & 0 & -49 & 0 & -1 \\
\hline 8 BEB & 2 & 2 & -1 & -1 & 2 & 0 & -15 & 0 & 0 \\
\hline 9 HFS & -10 & 0 & -3 & -1 & -6 & 0 & -20 & 0 & -1 \\
\hline $10 \mathrm{HFS}$ & -4 & -3 & -1 & 0 & -2 & 2 & 1 & 0 & 0 \\
\hline $11 \mathrm{HFS}$ & -5 & -3 & 1 & 0 & -2 & -1 & -38 & 1 & 1 \\
\hline $12 \mathrm{HFS}$ & 0 & 0 & 3 & -3 & 0 & 0 & -55 & 1 & 0 \\
\hline $13 \mathrm{HFS}$ & 14 & 8 & 6 & -1 & 0 & 1 & 6 & 0 & 0 \\
\hline $14 \mathrm{HFS}$ & -17 & -8 & -2 & -2 & -5 & 0 & -2 & 1 & 0 \\
\hline $15 \mathrm{HFS}$ & 0 & 1 & 0 & 0 & 0 & 1 & -12 & 0 & 0 \\
\hline
\end{tabular}

$\mathrm{a}-\mathrm{e}=$ the five subscales of the 24-item CDQ-24 QOL.

it is not possible to comment on whether the OP patients were more light-sensitive or had more light-induced spasm than $\mathrm{ON}$ patients, but this may have influenced response rates between the two groups.

Many questions remain unanswered about the mechanism of action of the BOF on reducing spasm, and these should be the subject of further study. Firstly, if reduction in light transmission is their main effect, why do they work with unilateral wear, and would we have expected a greater effect if they had been worn bilaterally, as tinted lenses are? Inducing visual blur bilaterally would be impractical and unacceptable to most patients. It has also been our experience that unilateral wear evokes a sufficient response in those patients responsive to the BOF, and that this response is the same regardless of the side of wear (left or right), even in our HFS patients. These observations suggest that the underlying mechanism for the BOF is much more complex than just reduction in light transmission or vision. It may well simply act as a distraction, but nevertheless, this possible distraction-effect remained for the duration of the study and further studies may help define long-term benefit.

We acknowledge significant flaws in this study. Confounding factors that may have influenced outcomes in the two groups include placebo effect, selection bias, and duration of wear and strength of BOF. As patients were aware of the working hypothesis on enrolment into the study, and were not masked to their occlusion status, a placebo effect in the OP group may have contributed to their better overall results.
The better results obtained in the OP groups may also have been due to selection bias, as $50 \%$ of the OP group (3/6) had already been using the BOF for blepharospasm with good effect prior to enrolment.

It would be expected that patients who wore the BOF more consistently, and who tolerated more reduction in visual acuity, would gain more effect. Whilst a greater proportion of the OP group $(80 \%)$ wore the BOF full-time compared to the ON group (67\%), and the mean strength of foil used was also stronger in the OP group (0.45) than in the ON group (0.58), the small numbers of patients in both groups did not allow meaningful statistical analysis of this difference. However, duration of wear and strength of BOF prescribed is patient-driven and reflects what is acceptable to patients in everyday life. Enforcing a certain strength and duration of wear between the OP and ON patients in this study may have increased the accuracy of our results, but would not have reflected how we would practically prescribe this treatment to our patients. The strength of BOF worn ranged between $0.3-0.6$ in the $\mathrm{OP}$ group, equating to a unilateral reduction of vision of 20/30-20/80 (mean 20/40). This did not affect patients' ability to carry out normal daily tasks; however, patients often opted not to use the BOF during activities such as work, where clear binocular vision was essential. In our study we excluded patients who were predominantly monocular, however, unilateral blur may contribute to loss of depth perception and impair mobility in patients who are already impaired, many of whom may also be elderly. This further limits the suitability of BOF or other uniocular occlusion modalities. 
With no conceivable cure in sight and current treatments offering variable symptomatic control, any treatment which is safe, effective, affordable and acceptable to patients, is a welcome addition to the evidence-based management of this difficult condition. The BOF meets many of these criteria. There were no significant side effects from wearing the BOF in this study, but practical problems, such as the accumulation of dirt and bubbles under the stick-on foil, which increased visual blur, occurred. This was easily rectified by changing of this cheap foil (approximately 4 US dollars each). Whilst one OP patient in this study has not required any BT since wearing the BOF, all other patients have required ongoing BT injections for optimal blepharospasm control. We anticipate that the impact of the BOF will be as an adjunctive to the mainstay treatments of BT and surgery. It remains to be seen whether the BOF can reduce the amount or frequency of BT required in responsive patients, but this would clearly be beneficial.

In conclusion, the results of this small pilot study suggest that patients with idiopathic blepharospasm or HFS may demonstrate the phenomenon of occlusion-positivity. Those positive are more likely to experience a reduction in spasm with a BOF than occlusion-negative patients. Whilst further study with larger patient numbers is required, we suggest that patients with BEB or HFS be examined for occlusion status, and that the BOF be considered as an adjunctive treatment in patients who are occlusion-positive.

\section{MEETING PRESENTATIONS}

Royal College of Ophthalmologist's Annual Congress, May 2008.

\section{REFERENCES}

[1] Tan N, Chan L, Tan E. Hemifacial spasm and involuntary facial movement. Q J Med 2002; 95: 493-500.

[2] Wang A, Jankovic J. Hemifacial spasm: clinical findings and treatment. Muscle Nerve 1998; 21: 1740-7.
[3] Anderson R, Patel B, Holds J, Jordan D. Blepharospasm: past present and future. Ophthal Plast Reconstr Surg 1998; 14: 305-17.

[4] O'Day J. Use of BT in neuro-ophthalmology. Curr Opin Ophthalmol 2001; 12: 419-22.

[5] Snir M, Weinberger D, Bouria D, Kristal-Shalit O, Dotan G, AxerSiegel R. Quantitative changes in BT: a treatment over time in patients with essential blepharospasm and idiopathic hemifacial spasm. Am J Ophthalmol 2003; 136: 99-105.

[6] Chapman K, Bartley G, Waller R, Hodge D. Follow-up of patients with essential blepharospasm who underwent eyelid protractor myectomy at the Mayo clinic from 1980 through 1995. Ophthal Plast Reconstr Surg 1999; 15: 106-10.

[7] Yen M, Anderson R. Orbicularis oculi muscle graft augmentation after protractor myectomy in blepharospasm. Ophthal Plast Reconst Surg 2003; 19: 287-96.

[8] Adams WH, Digre K, Patel B, Anderson R, Warner J, Katz B. The evaluation of light sensitivity in benign essential blepharospasm. Am J Ophthalmol 2006; 142: 82-7.

[9] Blackburn M, Digre K, Warner J, Katz B. FL-41 tint improves light sensitivity and eyelid spasms in patients with benign essential blepharospasm. $31^{\text {st }}$ Annual meeting of the North American NeuroOphthalmology Society. Copper Mountain, CO 2005.

[10] Herz N, Yen MT. Modulation of sensory photophobia in essential blepharospasm with chromatic lenses. Ophthalmology 2005; 112: 2208-11.

[11] Lamb R, Digre K, Smith G, Warner J, Nandedkar S, Katz B. Electromyographic evidence that FL-41 tinted spectacles decrease blink frequency and force in benign essential blepharospasm. $32^{\text {nd }}$ Annual meeting of the North American Neuro-ophthalmology Society. Tucson, Arizona 2006.

[12] Sagili S, Elston J, Malhotra R. Facial palsy-induced blepharospasm relieved by a Bangerter foil. Mov Disord 2005; 20: 1231-2.

[13] Muller J, Wissel J, Kemmler G, Voller B, Bodner T, Schneider G. Craniocervical dystonia questionnaire (CDQ-24): development and validation of a disease-specific quality of life instrument. J Neurol Neurosurg Psychiatry 2004; 75: 749-53.

[14] Vitale S, Miller N, Mejico L, et al. A randomised placebocontrolled crossover clinical trial of super blue-green algae in patients with essential blepharospasm or Meige syndrome. Am J Ophthalmol 2004; 138: 18-32.

[15] Hallett M. Blepharospasm: recent advances. Neurology 2002; 59: 1306-12.

[16] Cetinkaya A, Brannan PA. What is new in the era of focal dystonia treatment? botulinum injections and more. Curr Opin Ophthalmol 2007; 18: 424-9.

(C) Malhotra et al.; Licensee Bentham Open.

This is an open access article licensed under the terms of the Creative Commons Attribution Non-Commercial License (http: //creativecommons.org/licenses/by$\mathrm{nc} / 3.0 /$ ) which permits unrestricted, non-commercial use, distribution and reproduction in any medium, provided the work is properly cited. 